\title{
Forces between Hydrophobic Solids in Concentrated Aqueous Salt Solution
}

\author{
Dean J. Mastropietro and William A. Ducker* \\ Department of Chemical Engineering, Virginia Tech, Blacksburg Virginia, 24060, USA
}

(Received 4 October 2011; revised manuscript received 8 December 2011; published 5 March 2012; publisher error corrected

10 April 2012)

\begin{abstract}
Much research has focused on the discovery and description of long-ranged forces between hydrophobic solids immersed in water. Here we show that the force between high contact-angle solids in concentrated salt solution $(1 \mathrm{M} \mathrm{KCl})$ agrees very well with van der Waals forces calculated from Lifshitz theory for separations greater than $5 \mathrm{~nm}$. The hydrophobic solids are octadecyltrichlorosilane-coated glass, with an advancing contact angle of $108^{\circ}$. Thus, in $1 \mathrm{M}$ salt solution, it is unnecessary to invoke the presence of a hydrophobic force at separations greater than $5 \mathrm{~nm}$. Through measurement in salt solution, we avoid the necessity of accounting for large electrostatic forces that frequently occur in pure water and may obscure resolution of other forces.
\end{abstract}

DOI: 10.1103/PhysRevLett.108.106101

PACS numbers: 68.08.De, 34.20.Gj, 82.70.Dd

The "hydrophobic effect" is a term used to describe the high energy of hydration of apolar molecules in water that is responsible for the self-assembly of many biological molecules, surfactants and polymers in water. The high energy of hydration usually arises from the negative entropy of hydration. There remains an unsolved question: what are the interactions between larger-scale $(10 \mathrm{~nm}-$ macroscopic) hydrophobic materials?[1] This interaction potentially influences a range of phenomenon including nanoparticle or colloidal stability, adhesion, wetting, and froth flotation.

$\mathrm{X}$-ray diffraction and ellipsometry for isolated macroscopic hydrophobic surfaces both show that water structure is disturbed over only a very small distance: only the width of a few water molecules at most [2-5]. When water is confined in a thin film between two hydrophobic surfaces, the situation is qualitatively different because the existence of the thin water film can be thermodynamically unstable (depending on the geometry) and water may only exist in a kinetically trapped state [6]. However, simulation [7-10] predicts diminished density and attractive forces that extend over only a few nanometers at most.

However, there has been a long and well-documented scientific debate showing a very wide range of results of force-separation measurements for macroscopic hydrophobic solids. Some measurements have shown attractive forces with ranges up to $100 \mathrm{~nm}$ or more. A more complete picture is contained in several reviews [11,12]. The key issue is that all measurements of the force-separation relationship measure the total force, and the effect of hydrophobicity "the hydrophobic force" is obtained by subtraction of estimates of the other forces. It is now recognized that many earlier measurements included very strong influences from charge patches [13-15] and other electrostatic effects, or adsorbed nanobubbles [16], that were not accounted for in the original work. There have been very few experiments where the "real" effects of hydrophobicity can be distinguished. Two papers stand out.

Wood and Sharma [17] measured the force in deaerated water between $\mathrm{cm}$-sized mica surfaces coated in octadecyltriethoxysilane (OTE). They measured zero force at large separations, and a mechanical instability at about $17 \mathrm{~nm}$ separation; the mechanical instability indicated an attractive force with a gradient exceeding the stiffness of the measurement device, but the actual attractive force was not measured.

Meyer et al. [18] also measured the force between OTEcoated mica surfaces in water but without degassing the solution. Consistent with Sharma, they found that there was no force at separations greater than $15 \mathrm{~nm}$. The authors report an instability at a distance of about $13 \mathrm{~nm}$, but they determined the surface force in the unstable regime by an analysis of the dynamic motion of the surface attached to the measurement spring [19] assuming that the slip-length is zero. At separations less than about $15 \mathrm{~nm}$, they calculate an attractive force that is much greater $(\sim 100 \times)$ than the Lifshitz estimate of van der Waals forces. Cottin-Bizonne et al. [20] subsequently reported that the slip-length for a very similar hydrophobic solid is $15 \mathrm{~nm}$ per surface. Such a slip length would cause a large reduction in the estimated hydrodynamic force and a reduction in the hydrophobic force that is calculated from the data of Meyer et al. Although Meyer et al provide one of the best current estimates for the hydrophobic force, the estimate is strongly affected by an assumed parameter.

In this Letter we describe what we believe to be the first force measurements between hydrophobic surfaces that (a) show a net attractive force in a quasistatic measurement, and (b) are uncomplicated by estimates of forces other than the ubiquitous van der Waals force. Our selected system consists of smooth borosilicate-glass solids coated in polymerized octadecyltrichlorosilane (OTS) that are immersed in degassed aqueous $1 \mathrm{M}$ roasted $\mathrm{KCl}$ solution. 
The salt screens electrostatic forces, the degassing minimizes bubble formation, and the system has weak van der Waals forces so that hydrophobic forces are more easily discerned. Together, these conditions combine to enable us to measure the hydrophobic force.

Estimation of the hydrophobic force relies on accurate estimation of the van der Waals forces. We calculated theoretical van der Waals interactions per unit area for the planar 5-layer glass-OTS-aqueous salt-OTSglass system using Lifshitz theory [21] (see Fig. 1). We accounted for the effect of screening by the salt by adjusting the zero frequency term, $A_{0},[21]$ :

$$
A_{0}(\kappa) / A_{0}(0) \approx(1+2 D \kappa) e^{-2 D \kappa},
$$

where $\kappa^{-1}$ is the Debye length and $D$ is the separation. Microwave and UV absorption data for water were found in Parsegian [21]. Ultraviolet absorption parameters $C_{\mathrm{UV}}$ and $\omega_{\mathrm{UV}}$ for BK7 borosilicate glass were approximated using "Cauchy plots" as outlined in Hough \& White [22] Coefficients for borosilicate crown glass (BK7)[23,24] were used with Sellmeier's equation for creating the Cauchy plots. Optical data for the exact glass we used is not available, but an idea of the effect of changes in glass composition is seen from the force for pure $\mathrm{SiO}_{2}$ with a $2.7 \mathrm{~nm}$ hydrocarbon film, which is about $20 \%-30 \%$ smaller than for BK7 glass. Spectral data for octadecane (the alkane portion of OTS) could not be located so absorption parameters for hexadecane were used in the modeling [22]. The calculated force for a $2.7 \mathrm{~nm}$ layer of tetradecane and hexadecane were the same, so error in using hexadecane for octadecane should be small. In principle, the addition of salt also changes the UV contribution to the force, but $\mathrm{KCl}$ is not a very polarizable salt [25]. The effect of salt on the

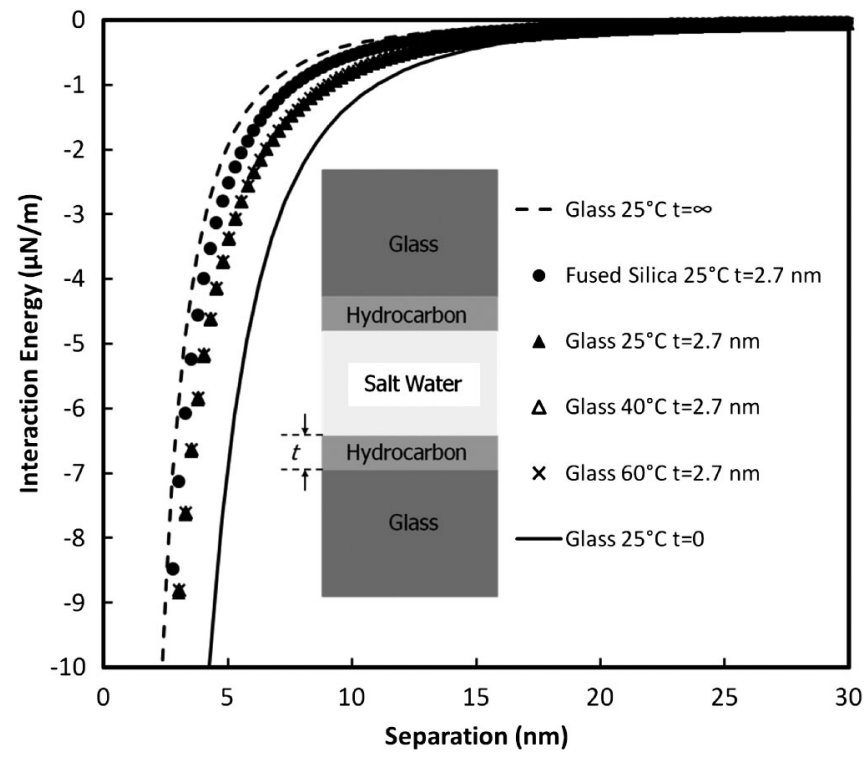

FIG. 1. Lifshitz calculation for van der Waals interactions between two semi-infinite half spaces coated in a layer of hexadecane across $1 \mathrm{M}$ aqueous salt solutions.
UV contribution to the van der Waals force of $1.0 \mathrm{M} \mathrm{KCl}$ was examined by measuring the refractive index as a function of wavelength in the range 400-700 nm and using a "Cauchy plot" to determine $C_{\mathrm{UV}}$ and $\omega_{\mathrm{UV}}$ for the $1.0 \mathrm{M}$ $\mathrm{KCl}$ solution. The effect on the van der Waals force was insignificant. More significant errors in the calculated van der Waals force arise from errors in the thickness of the film, and properties of the glass. Figure 1. shows that even for the extreme limits of zero alkane thickness or infinite thickness of alkane, the force increases or decreased by only a factor of 2 . A more realistic error in film thickness of $0.4 \mathrm{~nm}$ propagates into an error in van der Waals force of about 5\%. The important point is that the van der Waals forces are quite weak, independent of temperature, and fall in a relatively narrow range even when allowing substantial variation in film thickness or solid composition.

Force-distance data were obtained from measurements using an MFP-3D atomic force microscope (Asylum Research, Santa Barbara), which measures cantilever deflection (and thus force) as a function of displacement by a piezoelectric translation device at a rate of $50 \mathrm{kHz}$. Forces were measured between a $20 \mu \mathrm{m}$ glass sphere and a glass plate, each coated with a self-assembled monolayer of octadecyltrichlorosilane (OTS) in a large reservoir $(\sim 2 \mathrm{~mL})$ of $1.0 \mathrm{M} \mathrm{KCl}$. During measurement, the volume of the chamber decreases by about $10^{-6} \mathrm{~mL}$ for every $\mathrm{nm}$ decrease in separation, but the presence of air in the chamber limits the increase in pressure. In any case, the seal on the chamber allows the exit of air when the pressure is a little over $1 \mathrm{~atm}$. Force-separation plots were obtained using the analysis method outlined in Ducker et al. [26,27]. with the following variations: (i) the translation was measured using a linear variable differential translation sensor (LVDT) and (ii) virtual deflection was removed from the raw deflection voltage-LVDT signal [28]. There was negligible effect of the virtual deflection correction because the LVDT range was so small. The stiffness of each cantilever was measured by the thermal method [29], and ranged from $0.49-0.77 \mathrm{~N} / \mathrm{m}$.

Aqueous salt solutions were prepared by combining Milli- $Q$ water with roasted $\mathrm{KCl}$, and degassed by three freeze-pump $(\sim 0.1 \mathrm{~mm} \mathrm{Hg})$-thaw cycles. Degassed solutions were used to decrease the likelihood of nanobubble nucleation at the solid-liquid interface. Glass spheres were purchased from Duke Scientific Corporation and glass plates were Fischer Finest cover slips. Well-anchored OTS monolayers were used to produce hydrophobic solids. OTS coatings were prepared by submerging $\mathrm{O}_{2}$-plasma (250 mTorr, $2 \mathrm{~min}, 100 \mathrm{~W}$ ) treated glass plates or cantilevers with mounted spheres in 3-5 mM OTS in hexadecane solutions overnight ( $\sim 15$ hours). OTS-coated films were dipped in fresh chloroform to remove excess solvent and unattached OTS to produce smooth, uniform monolayers free of asperities. OTS monolayers prepared in this 
manner were found to have advancing angles of $108^{\circ}$ and receding contact angles of $95^{\circ}$ in $1.0 \mathrm{M} \mathrm{KCl}$ solution and angles of $107^{\circ}$ and $95^{\circ}$ in pure water. Contact mode AFM scans showed an RMS roughness of $<0.5 \mathrm{~nm}$ for plates over $20 \mu \mathrm{m}^{2}$ and $<1.0 \mathrm{~nm}$ for spheres over $1 \mu \mathrm{m}^{2}$, while ellipsometry measured a thickness of approximately $2.7 \mathrm{~nm}$, assuming a dielectric constant of 2.40 at $633 \mathrm{~nm}$ for the hydrocarbon layer. After exposure to $1.0 \mathrm{M} \mathrm{KCl}$ for $3 \mathrm{~h}$ (the maximum duration of the experiment), the advancing and receding contact angle for the $1.0 \mathrm{M} \mathrm{KCl}$ solution were $109 \pm 1^{\circ}$ and $95 \pm 3^{\circ}$ respectively, and the roughness was unchanged.

Surface force measurements show the attractive force between the hydrophobic surfaces (Fig. 2). The measurements are noisy because thermal noise is similar in amplitude to the de force for separations greater than about $10 \mathrm{~nm}$. A 101-point average $(2 \mathrm{~ms})$ removes much of the noise and reveals the net attraction at separations less than about $12 \mathrm{~nm}$. When the gradient of the surface force exceeds the spring constant, the sphere position is mechanically unstable. This occurs at about $6 \mathrm{~nm}$ in Fig. 2,

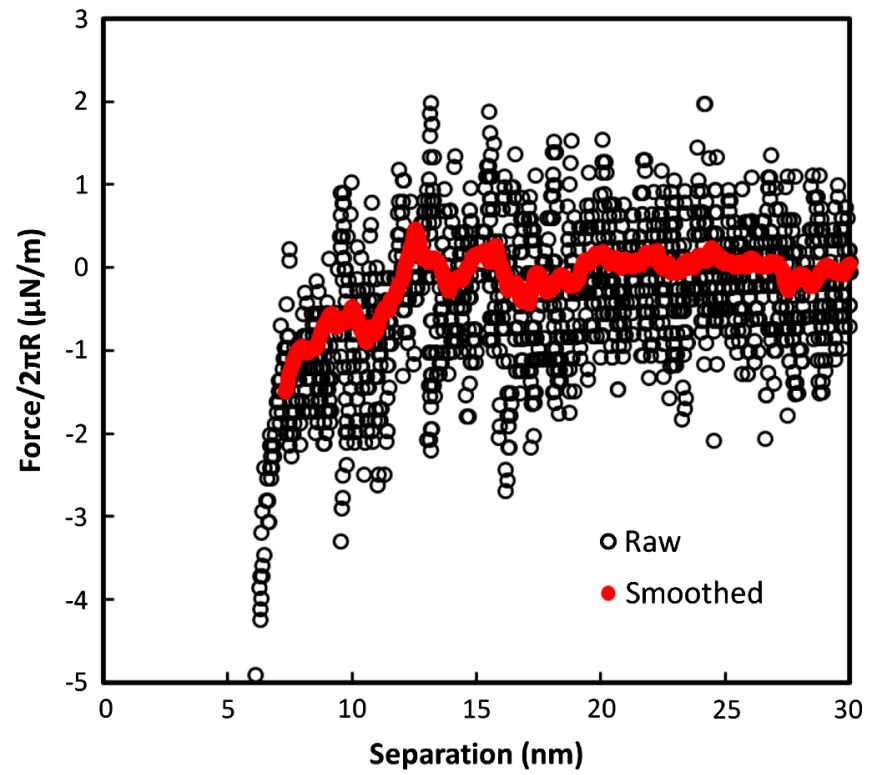

FIG. 2 (color online). Measured force vs separation for OTScoated borosilicate glass interacting across aqueous $1.0 \mathrm{M} \mathrm{KCl}$ solution. The force has been normalized by $2 \pi R$, which for a sphere-plate geometry is equal to the energy per unit area for flat plates (the Derjaguin approximation) [34]. Open circles show measured points at separations greater than the point of mechanical instability for the sphere (which occurs when the gradient of the attractive force equals the spring constant). The red (filled) circles represent smoothed data: the raw deflection data was smoothed using a 101-point moving average before conversion to force-distance. The smoothed curve was truncated 50 points $(\sim 0.50 \mathrm{~nm})$ before the mechanical instability. In this data the mechanical instability occurred at about $6 \mathrm{~nm}$, and in a series of repeat runs, instabilities occurred at 8.5, 10.9, 6.5, 6.7, $7.5,6.8,6.2,6.2,6.7$, and $7.0 \mathrm{~nm}$. and it is not possible to make equilibrium measurements at smaller separations.

Figure 3 shows results in which data from several approaches are averaged into $0.5 \mathrm{~nm}$ bins to reduce noise. Results for two different experiments are shown. At small separations $(<6 \mathrm{~nm})$ there are too few data points for averaging and the gradient is large, so individual measurements are shown. This averaged data is shown together with the calculated van der Waals force from Fig. 1 for the $2.7 \mathrm{~nm}$ hydrocarbon film. Extreme limits of the calculated force for zero and infinite thickness films are also shown.

The measured force agrees very well with the Lifshitz calculation for separations greater than $6 \mathrm{~nm}$. From 5-6 nm the fit is worse, but also the error is greater for both the Lifshitz theory (large effect of material properties and thickness) and the measured force (few measured points). Note also that there is a significant error in comparing the zero of separation for the theory and the experiment. The theory assumes a mathematical plane between layers whereas the real materials have combined rms roughness of $1.5 \mathrm{~nm}$. Perhaps the limiting difficulty in comparing the theory and experiment is the unknown effect of asperities

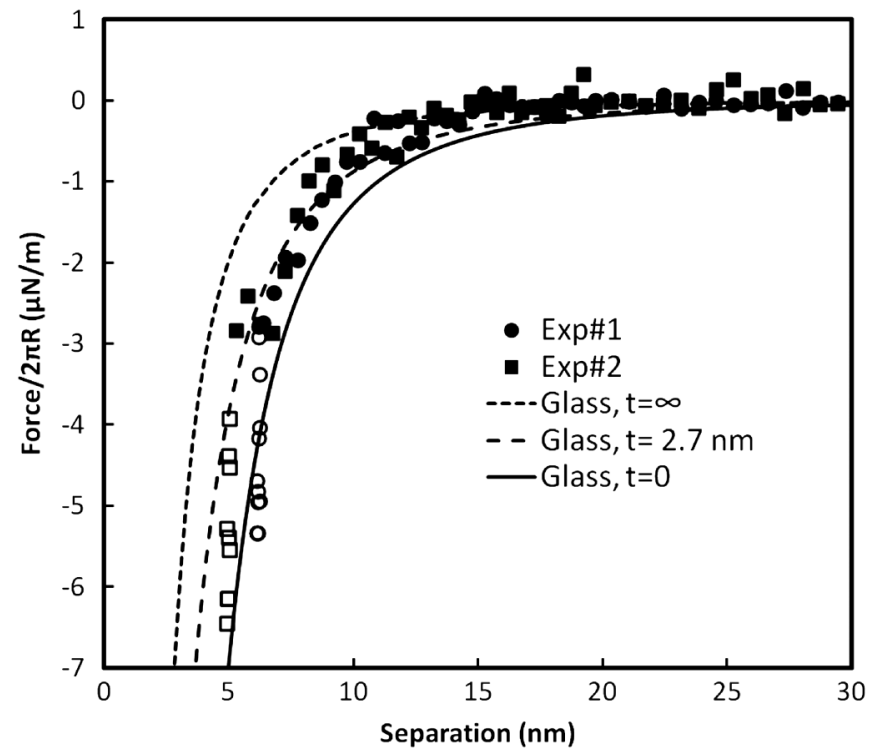

FIG. 3. Comparison between measured forces and Lifshitz calculation for borosilicate glass coated in OTS immersed in $1 \mathrm{M}$ aqueous salt at $23^{\circ} \mathrm{C}$. Circles and Squares represent data from different experiments. Lifshitz calculations represent the limiting cases of infinite and zero hydrocarbon thickness as well as the measured thickness of $2.7 \mathrm{~nm}$. Closed symbols represent data averaged from several force runs. The random error in the force is about $\pm 0.06 \mu \mathrm{N} / \mathrm{m}$ (i.e. about the point size) and in separation is $\pm 0.1 \mathrm{~nm}$. There is a systematic error of $\pm 20 \%$ in the force arising from errors in the spring constant, the radius and the calibration of the spring deflection ("invols"). At small separations, there are fewer data points, so the data is not averaged (shown as open symbols). The measured interactions agree with the calculated van der Waals interaction at separation greater than about $6 \mathrm{~nm}$. 


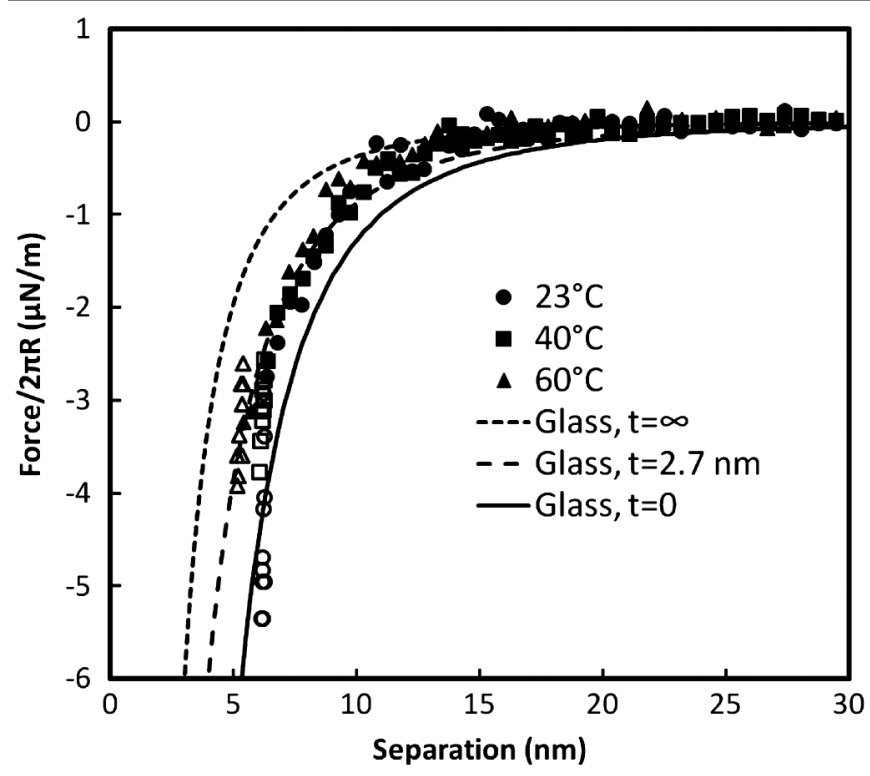

FIG. 4. Force as a function of temperature for OTS-coated borosilicate glass interacting across $1.0 \mathrm{M} \mathrm{KCl}$. The measurements cannot resolve differences as a function of temperature. Filled markers represent averaged data points while open markers represent single measured data points.

on the surfaces, which we estimate could cause a shift between experimental and theoretical data of up to $1 \mathrm{~nm}$. From Fig. 3 this has a large effect on the data in the range 5-7 $\mathrm{nm}$. The combined effect of all these errors can be seen by comparing two independent experiments in Fig. 3: the two data sets are very similar, but shifted by about $1 \mathrm{~nm}$ in separation. Considering these errors, the agreement between van der Waals force and measurement for salt solution in the range 5-6 nm is good, the agreement is much better than the $\sim 100 \times$ discrepancy reported in pure water [18]. Given the good agreement, it seems unnecessary at this point to invoke the existence of an additional theoretical force to describe the results. In other words, the hydrophobic force in $1.0 \mathrm{M}$ salt at separations greater than $5 \mathrm{~nm}$ is, at most, a correction of similar magnitude to the error in the van der Waals force, and perhaps zero.

The functional form and magnitude of the measured force suggest that the force can be explained entirely in terms of Lifshitz theory. Another approach to understanding the force between hydrophobic surfaces is to examine its dependence on temperature. The term "hydrophobicity" is usually associated with an increase in entropy arising from changes in water structure. The entropic contribution to the attractive force can be extracted from measurements of force as a function of temperature at fixed separation, $D$, and pressure, $P$,

$$
\left(\frac{\partial \Delta G}{\partial T}\right)_{D, P}=-\Delta S,
$$

where $\Delta G$ is the difference between the Gibbs free energy of the film at separation, $D$, and infinity and $\Delta S$ is the difference in the entropy of the film at separation $D$ and infinity.

Forces between hydrophobic solids in $1.0 \mathrm{M} \mathrm{KCl}$ as a function of temperature are shown in Fig. 4. We cannot resolve differences in force over the range $23-60{ }^{\circ} \mathrm{C}$ and therefore we conclude that the force has only small entropic contributions. Thus it is difficult to associate the measured force with changes in water structure as a film thins.

In contrast to measurements at separations greater than $5 \mathrm{~nm}$, measurements of the pull-off force (force to separate surfaces from contact) do show a small temperature dependence, as shown in Table I. The force decreases with temperature, as expected for (predominantly entropic) hydrophobic effect [31,32], which describes molecular-scale hydration of hydrocarbons. Note that there is a very large error associated with Lifshitz theory at zero separation because (a) our implementation does not account for film roughness and (b) uncertainty in how to treat van der Waals forces at small separations [33]. JKR theory can be used to estimate the solid-liquid surface tension, which is about $20 \mathrm{mJm}^{-2}$. This is lower than expected for a hydrocarbonwater interface $\left(\sim 50 \mathrm{mJm}^{-2}\right)$, but not surprising for surfaces with nanometer-scale roughness.

In conclusion, the measured force between smooth hydrophobic solids $\left(\theta_{\text {adv }}=108^{\circ}, \theta_{\text {rec }}=95^{\circ}\right)$ in degassed concentrated aqueous salt solution $(1 \mathrm{M} \mathrm{KCl})$ agrees with the van der Waals force calculated from Lifshitz theory for separations greater than $5 \mathrm{~nm}$. Measurements at smaller separations were complicated by mechanical instabilities in the spring so are not discussed here. The good agreement between the measured force and the calculated van der Waals force renders it unnecessary to invoke the existence

TABLE I. Pull-off force

\begin{tabular}{lcccc}
\hline \hline $\begin{array}{l}\text { Temperature } \\
{ }^{\circ} \mathrm{C}\end{array}$ & $\begin{array}{c}\text { Energy per area } \\
\left(\mathrm{mJ} / \mathrm{m}^{2}\right)\end{array}$ & $\begin{array}{c}\text { Lifshitz energy }^{\mathrm{c}} \\
(S=1.0 \mathrm{~nm})\left(\mathrm{mJ} / \mathrm{m}^{2}\right)\end{array}$ & $\begin{array}{c}\text { Lifshitz energy }^{\mathrm{c}} \\
(S=0.5 \mathrm{~nm})\left(\mathrm{mJ} / \mathrm{m}^{2}\right)\end{array}$ & $\begin{array}{c}\gamma_{\mathrm{sl}}(\mathrm{JKR})^{\mathrm{b}} \\
\left(\mathrm{mJ} / \mathrm{m}^{2}\right)\end{array}$ \\
\hline 23 & $37.1 \pm 0.6$ & 0.07 & 0.30 & $24.7 \pm 0.4$ \\
40 & $34.9 \pm 0.2$ & 0.07 & 0.30 & $23.2 \pm 0.2$ \\
60 & $30.5 \pm 0.2$ & 0.07 & 0.30 & $20.3 \pm 0.1$ \\
\hline \hline
\end{tabular}

a+ calculated from $F / 2 \pi R$.

${ }^{\mathrm{b}}$ From JKR theory from $\gamma_{\mathrm{SL}}=F / 3 \pi R$ [30].

${ }^{\mathrm{c}}$ Lifshitz energy is the energy for two surfaces. 
of a long-range "hydrophobic force" that extends beyond $5 \mathrm{~nm}$ in concentrated salt solution. Furthermore, the lack of measurable variation in the force with temperature in the range $25-60^{\circ} \mathrm{C}$ is consistent with an enthalpic force, and at odds with customary descriptions of hydrophobicity that are based on changes in water structure.

Compared to measurement in pure water, measurements in salt $(1 \mathrm{M} \mathrm{KCl})$ have relevance to interactions in sea water $\left(\sim 0.5 \mathrm{M} \mathrm{Cl}^{-}\right)$and biological conditions $(\sim 0.15 \mathrm{M}$ $\mathrm{Cl}^{-}$), and also screens electrostatic forces, thereby removing ambiguity in whether to attribute forces to waterstructural or electrostatic origins. The addition of salt and the removal of gas may affect water structure, so these results cannot be used to exclude the possibility that a longrange hydrophobic force does exist in pure water with an equilibrium concentration of dissolved gas. However, arguments for a long-range force in water would need to be based on differences in water structure that occur as a result of dissolved salt or gas.

The authors acknowledge useful discussions with RoeHoan Yoon, Jan-Christer Eriksson, Alenka Luzar, and Antonio Checco, assistance with the calculation of van der Waals forces from Shunxi $\mathrm{Ji}$, and funding from Virginia Tech.

*wducker@vt.edu

[1] K. Lum, D. Chandler, and J. D. Weeks, J. Phys. Chem. B 103, 4570 (1999).

[2] T. Jensen, M. Østergaard Jensen, N. Reitzel, K. Balashev, G. Peters, K. Kjaer, and T. Bjørnholm, Phys. Rev. Lett. 90, 086101 (2003).

[3] M. Mezger, H. Reichert, S. Schöder, J. Okasinski, H. Schröder, H. Dosch, D. Palms, J. Ralston, and V. Honkimäki, Proc. Natl. Acad. Sci. U.S.A. 103, 18401 (2006).

[4] A. Poynor, L. Hong, I. K. Robinson, S. Granick, Z. Zhang, and P. A. Fenter, Phys. Rev. Lett. 97, 266101 (2006).

[5] M. Mao, J.H. Zhang, R.H. Yoon, and W. A. Ducker, Langmuir 20, 1843 (2004).

[6] K. Leung, A. Luzar, and D. Bratko, Phys. Rev. Lett. 90, 065502 (2003).

[7] D. Bratko and A. Luzar, Langmuir 24, 1247 (2008).

[8] A. Luzar, D. Bratko, and L. Blum, J. Chem. Phys. 86, 2955 (1987).

[9] A. J. Pertsin, T. Hayashi, and M. Grunze, J. Phys. Chem. B 106, 12274 (2002).

[10] T. Hayashi, A. J. Pertsin, and M. Grunze, J. Chem. Phys. 117, 6271 (2002).
[11] H. K. Christenson and P. M. Claesson, Adv. Colloid Interface Sci. 91, 391 (2001).

[12] M. U. Hammer, T.H. Anderson, A. Chaimovich, M. S. Shell, and J. Israelachvili, Faraday Discuss. 146, 299 (2010).

[13] J. Zhang, R.H. Yoon, M. Mao, and W.A. Ducker, Langmuir 21, 5831 (2005).

[14] E. E. Meyer, Q. Lin, T. Hassenkam, E. Oroudjev, and J. N. Israelachvili, Proc. Natl. Acad. Sci. U.S.A. 102, 6839 (2005).

[15] S. Perkin, N. Kampf, and J. Klein, Phys. Rev. Lett. 96 (2006).

[16] P. Attard, Adv. Colloid Interface Sci. 104, 75 (2003).

[17] J. Wood and R. Sharma, Langmuir 11, 4797 (1995).

[18] E. E. Meyer, K. J. Rosenberg, and J. Israelachvili, Proc. Natl. Acad. Sci. U.S.A. 103, 15739 (2006).

[19] Q. Lin, E. E. Meyer, M. Tadmor, J. N. Israelachvili, and T. L. Kuhl, Langmuir 21, 251 (2005).

[20] C. Cottin-Bizonne, A. Steinberger, B. Cross, O. Raccurt, and E. Charlaix, Langmuir 24, 1165 (2008).

[21] V. A. Parsegian, Van Der Waals Force: A Handbook for Biologists, Chemists, Engineers, and Physicists (Cambridge University Press, Cambridge, England, 2006).

[22] D. B. Hough and L. R. White, Adv. Colloid Interface Sci. 14, 3 (1980).

[23] http://www.cvimellesgriot.com/products/Documents/ Catalog/Dispersion_Equations.pdf. Accessed: September 25th, 2011.

[24] M.A. Bevan and D.C. Prieve, Langmuir 15, 7925 (1999).

[25] H. I. Petrache, T. Zemb, L. Belloni, and V. A. Parsegian, Proc. Natl. Acad. Sci. U.S.A. 103, 7982 (2006).

[26] W. A. Ducker, T. J. Senden, and R. M. Pashley, Langmuir 8, 1831 (1992).

[27] W. A. Ducker, T. J. Senden, and R. M. Pashley, Nature (London) 353, 239 (1991).

[28] C. D. F. Honig and W. A. Ducker, J. Phys. Chem. C 111, 16300 (2007).

[29] J. L. Hutter and J. Bechhoefer, Rev. Sci. Instrum. 64, 1868 (1993).

[30] K. L. Johnson, K. Kendall, and A. D. Roberts, Proc. R. Soc. A 324, 301 (1971).

[31] C. Tanford, The Hydrophobic Effect: Formation of Micelles and Biological Membranes (Wiley, New York, 1973).

[32] J. N. Israelachvili, Intermolecular and Surface Forces (Academic Press, Amsterdam, 2011).

[33] L. R. White, J. Colloid Interface Sci. 343, 338 (2010).

[34] B. V. Derjaguin, Kolloid Z., 69, 155 (1934). See also, R. J. Hunter, Foundations, of Colloid Science (Oxford Science Press, Oxford, 1987), Vol. I, p. 191. 